\title{
NOUVELLE MÉTHODE DE MARQUAGE VITAL D'GUFS DE SALMONIDÉS PAR INCORPORATION OSMOTIQUE DE TÉTRACYCLINE A LA FÉCONDATION : EXPÉRIENCES PRÉLIMINAIRES SUR DES GUFS DE TRUITE FARIO (SALMO TRUTTA FARIO) ET DE TRUITES ARC-EN-CIEL (ONCORHYNCHUS MYKISS)
}

\author{
C. RUHLE (1), Colette GRIEDER (2)
}

(1) Service Chasse et Pêche du canton de Saint-Gall, CH-9001 SAINT-GALL, Suisse.

(2) Institut fédéral pour l'aménagement, l'épuration et la protection des eaux, $\mathrm{CH}-6047$ KASTANIENBAUM, Suisse.

\section{RÉSUMÉ}

Une méthode a été développée pour marquer les œufs de truites par de la tétracycline au moment de leur fécondation. La tétracycline mélangée à l'eau qui s'infiltre par osmose dans les œufs au moment de la fécondation ("swelling water") y persiste jusqu'au début de la calcification des otolithes. La substance fluorescente est incorporée dans l'otolithe en formation et y laisse ainsi une marque reconnaissable. Cette méthode est comparée à celle par balnéation des œufs embryonnés dans une solution de tétracycline.

\section{NEW METHOD FOR THE MARKING OF TROUT (SALMO TRUTTA FARIO AND ONCORHYNCHUS MYKISS) EGGS BY OSMOTIC INCORPORATION OF TETRACYCLINE AT FERTILIZATION}

\section{SUMMARY}

A method for marking trout eggs at the time of fertilization was found. The tetracycline added to the swelling water was absorbed and remained in the eggs until the otoliths started to calcify. It was then deposited in the growing otoliths and left a recognizable mark. This method is compared to the one applied to eyed eggs immersed in a tetracycline solution.

\section{INTRODUCTION}

Les expériences de marquage sont limitées à des poissons dont la taille permet l'application de marques sans leur porter préjudice et sans influencer leur comportement. En général, la grandeur des marques externes à disposition ne permet pas de marquer de très petits individus. Les informations obtenues par des expériences de marquage faites avec des estivaux ou des adultes ne peuvent pas toujours être extrapolées aux sujets de plus petite taille. Il peut donc s'avérer nécessaire de pouvoir marquer de très petits poissons ou même des larves. De telles opérations s'imposent pour des études de dynamique de population et surtout pour évaluer les conséquences sur le stock des immersions de larves, d'alevins et d'estivaux. Par exemple, dans l'aménagement des lacs d'Europe centrale, des informations sur les effets des immersions de corégones faites en général avec des larves (10-15 mm) pourraient être d'une grande importance économique. La taille des poissons pouvant être marqués a sensiblement pu être réduite lors de la mise sur le marché de marques magnétiques à implanter dans le cartilage du nez (coded-wire). Cependant, cette méthode ne peut pas être appliquée à des poissons de moins de $3 \mathrm{~cm}$. Pour le marquage de larves et d'alevins, il faut recourir aux marqueurs chimiques externes: spray (HENNICK et TYLER, 1970) ou internes: marqueurs vitaux )MEUNIER, 1972 et 1974) et métaux lourds (FRY et al., 1960).

Différents auteurs ont démontré les possibilités de marquage de poissons par les fluorochromes qui laissent des marques facilement reconnaissables sur les organes en cours de calcification. Les uns ont appliqué les fluoromarqueurs à dose précise relativement concentrée par injection (KOBAYASHI et al., 1964 ; JONES 1969 ; MEUNIER et BOIVIN, 1972, 1974 et 1978; MEUNIER, 1982); ce qui empèche l'application de cette méthode sur des poissons petits et très fragiles. Les autres (WEBER et RIDGWAY, 1962 ; JONES, 1969 ; TROJNAR, 1973 ; ODENSE et LOGAN, 1974; NAGIEC et NAGIEC, 1983 ; NAGIEC et al., 1983 ont introduit des fluoromarqueurs par ingestion en 
faible quantité mais à plusieurs reprises pour atteindre la dose optimale. Ceci peut se taire sur des poissons de toutes tailles, mais le contrôle du dosage du marqueur est difficile et le marquage irrégulier car l'incorporation du fluorochrome à la nourriture ainsi que la prise de nourriture sont variables.

En cherchant un autre moyen pour infiltrer une substance de marquage dans le corps du poisson, nous avons émis l'hypothèse que l'eau ("swelling water") s'infiltrant par osmose dans les cufs de Salmonidés, au moment de la fécondation, pourrait servir de voie de transport. Cette infiltration exigerait un stockage et un maintien des propriétés chimiques du marqueur jusqu'à la formation des premiers tissus osseux et des concrétions calcaires (otolithes). Le marqueur serait alors stocké dans le liquide périvitellin ou dans le vitellus pendant le développement embryonnaire pour être ensuite incorporé aux concrétions calcaires dès le début de leur minéralisation.

Nos expériences, basées sur cette hypothèse, ont commencé en 1979. Ayant mis en doute I'hypothèse que le marqueur serait stocké jusqu'au début de la minéralisation et n'ayant pas encore mis au point la méthode de détection de la marque, nous avons suspendu ces expériences jusqu'à la parution dès 1984, de publications concernant le marquage à la tétracycline par balnéation de larves et d'œufs embryonnés (HETTLER, 1984 ; SCHMITT, 1984 ; TSUKAMOTO, 1985 ; DABROWSKI et TSUKAMOTO, 1986 ; LORSON et MUDRAK, 1987 ; NAGIEC et al., 1988). Ces nouvelles données nous ont incités à reprendre nos recherches.

\section{MATÉRIEL ET MÉTHOdES}

L'idée de transférer de la tétracycline comme substance marquante avec l'eau permettant aux œufs de se gonfler a été appliquée sur des œufs de truite fario (Salmo trutta fario) et de truites arc-en-ciel (Oncorhynchus mykiss).

Les œufs et la laitance ont été prélevés sur des poissons anesthésiés dans une solution de MS 222 Sandoz ( $1: 15.000)$. Avant ce prélèvement, les individus ainsi tranquillisés ont été soigneusement essuyés. Après mélange des œufs et de la laitance, les œufs ont été séparés en plusieurs lots ( 1.000 œufs de truites fario et $2.500 œ u f s$ de truites arc-en-ciel par concentration) dont chacun a été traité dans une solution de tétracycline (hydrochloride de tétracycline $\mathrm{C}_{22} \mathrm{H}_{24} \mathrm{~N}_{2} \mathrm{O}_{8} \mathrm{HCl}$ purum Fluka $98 \%(\mathrm{Cl})$ à différentes concentrations (tableau I). Après une heure, les œufs ont été rincés et placés sur des grilles d'incubation. Les alevins ont été élevés dans des bassins circulaires. Durant la période d'incubation et d'élevage, les pertes ont été enregistrées quotidiennement et des échantillons ont été prélevés 188, 199 et 226 jours après le marquage pour les truites fario et 118,128 et 155 jours après le marquage pour les truites arc-en-ciel.

A titre de comparaison, la méthode décrite par TSUKAMOTO (1985) ainsi que par DABROWSKI et TSUKAMOTO (1986) a été appliquée sur des œufs embryonnés de truite arc-enciel. Deux lots de $1.000 œ u f s$ chacuns ont été exposés à une solution de tétracycline de $600 \mathrm{mg} / \mathrm{l}$ à $5^{\circ} \mathrm{C}$, l'un pendant 14 heures et l'autre pendant 24 heures. Un troisième lot servait de contrôle. Des échantillons ont été pris $55,124,143,159$ et 202 jours après la balnéation.

Les otolithes ont été prélevés sur les divers échantillons. Ils ont été nettoyés, séchés, puis montés sur une lame dans une goutte de Powabond 101 ou 102 (colle au cyanoacrylate, Double $H$ International Ltd, Staines, Middlesex TW $184 \times$ Q). Les préparations sont durcies pendant 15 à 30 minutes à $60^{\circ} \mathrm{C}$. Les otolithes sont observés au microscope pour une première observation. Si le centre de l'otolithe est visible, l'observation peut se poursuivre en fluorescence. Si l'otolithe est trop épais pour voir le centre, il est aminci, par ponçage au papier abrasif P600 mouillé, jusqu'à ce que le centre soit atteint. La préparation une fois séchée est ensuite montée à l'Entellan (Merck) et couverte d'une lamelle. L'observation de la marque fluorescente se fait sous un microscope à fluorescence en lumière réfléchie, équipé d'un filtre à exitation bleue (standard Zeiss, filtre BP 450-490, FT510, barrière LP520, objectif $10 x$ et $20 x$ ). Sur une moyenne de 10 poissons, le prélèvement et l'examen des otolithes dure environ 20 minutes par spécimen.

\section{REsultats}

Les taux de mortalité enregistrés au cours de l'incubation et de l'élevage des lots de truites fario et arc-en-ciel traités au moment de la fécondation avec des solutions de différentes concentrations de tétracycline sont résumés dans les figures 1 et 2 ainsi que dans le tableau I; ce dernier renferme également les taux de mortalité des œufs embryonnés traités par balnéation pendant 14 ou 24 heures.

Pour les truites fario traitées au moment de la fécondation les taux de mortalité sont de 3.6 à $11.6 \%$ au moment de l'éclosion pour les solutions de tétracycline de $0.01 \mathrm{~g} / \mathrm{l}$ à $1 \mathrm{~g} / \mathrm{l}$ et de $9,1 \%$ pour les poissons non traités. A la fin des expériences ( 173 jours après la fécondaticn) ces taux sont, pour les mêmes concentrations, de $18.4 \%$ et $43.7 \%$. Les truites fario non traitées (contrôles) ont au même moment, un taux de mortalité de $25.9 \%$.

Pour les truites arc-en-ciel traitées de la même façon mais avec des solutions de tétracycline de $0.125 \mathrm{~g} / \mathrm{l}$ à $2 \mathrm{~g} / \mathrm{l}$ les taux de mortalité observés jusqu'au moment de l'éclosion sont de $46.4 \%$ et 
49.3\%. La solution de $10 \mathrm{~g} / \mathrm{l}$ a causé une mortalité subite et totale. La mortalité à l'éclosion des truites non traitées est de $25.4 \%$. A la fin de l'expérience (125 jours après la fécondation), ces taux sont de $55.6 \%$ et $50.8 \%$ pour ces mêmes concentrations. Les poissons non traités (contrôles) ont un taux de mortalité de $30.9 \%$.

Tableau I : Concentration des solutions de tétracycline appliquées dans les deux méthodes comparées, taux de mortalité jusqu'au moment de l'éclosion et pourcentage de poissons marqués.

Table I : Concentrations of tetracycline solutions used in the two methods being compared, mortality rates until hatching time and percentage of fish marked.

\begin{tabular}{lcccc}
\hline $\begin{array}{l}\text { Méthode } \\
\text { d'application et } \\
\text { espèce }\end{array}$ & $\begin{array}{l}\text { Concentration } \\
\text { de la solution }\end{array}$ & $\begin{array}{c}\text { Taux de } \\
\text { mortalité }\end{array}$ & $\begin{array}{c}\text { Nombre de } \\
\text { jours entre } \\
\text { marquage et } \\
\text { prélèvement }\end{array}$ & $\begin{array}{c}\text { Pourcentage de } \\
\text { poissons marqués }\end{array}$ \\
\hline
\end{tabular}

application au

moment de la

fécondation

$1979 / 1980$

truites fario

0 (contrôle)
1
1
1
0.2
0.001

9.1

188

0

11.6

188

199

60

11.6

226

$\begin{array}{rr}11.6 & 226 \\ 8.1 & 188\end{array}$

82

30

3.6

truites

0 (contrôle)

25.4

arc-en-ciel

$$
10
$$$$
100
$$

2

47.2

118

50

$2 \quad 47,2$

128

100

47.2

155

70

1

46.4

50

0.5

47.8

0.25

48.9

(1)

0.167

48.6

0.125

49.3

\section{application à}

\section{des cufs}

embryonnés

1987
truites
0 (contrôle)
$0.6 / 14 \mathrm{~h}$
$0.6 / 24 \mathrm{~h}$
$0.6 / 24 \mathrm{~h}$
(1) pas de vérification
(2) tous morts au début de l'expérience

32

15

55

124

15

15

143

159

15

202
0
0
0
28
45


truites fario marquées à la fécondation pertes pendant l'incubation et l'élevage 100 pertes en $\%$

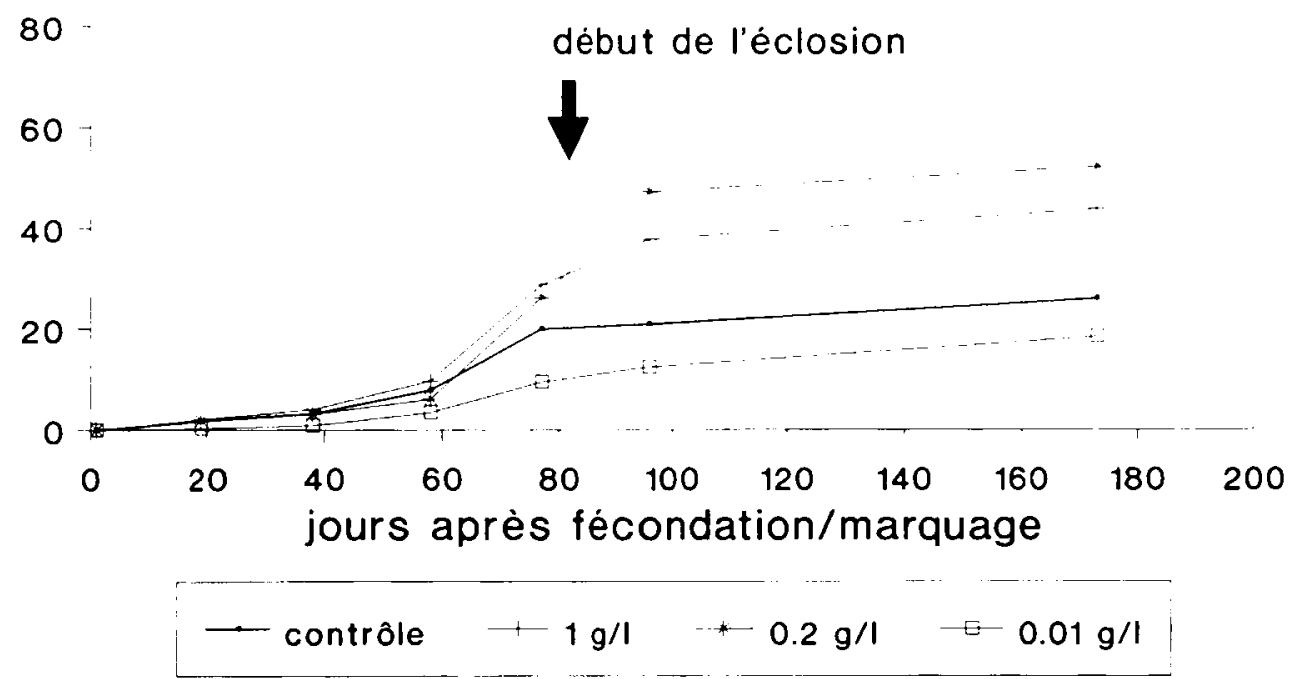

figure 1 : Mortalité pendant l'incubation et l'élevage de truites fario marquées à la tétracycline au moment de la fécondation à différentes concentrations.

figure 1 : Mortality during incubation and hatching of brown trouts marked at fertilisation with tetracycline at different concentrations.

truites arc-en-ciel marquées à la fécondation pertes pendant l'incubation et l'élevage

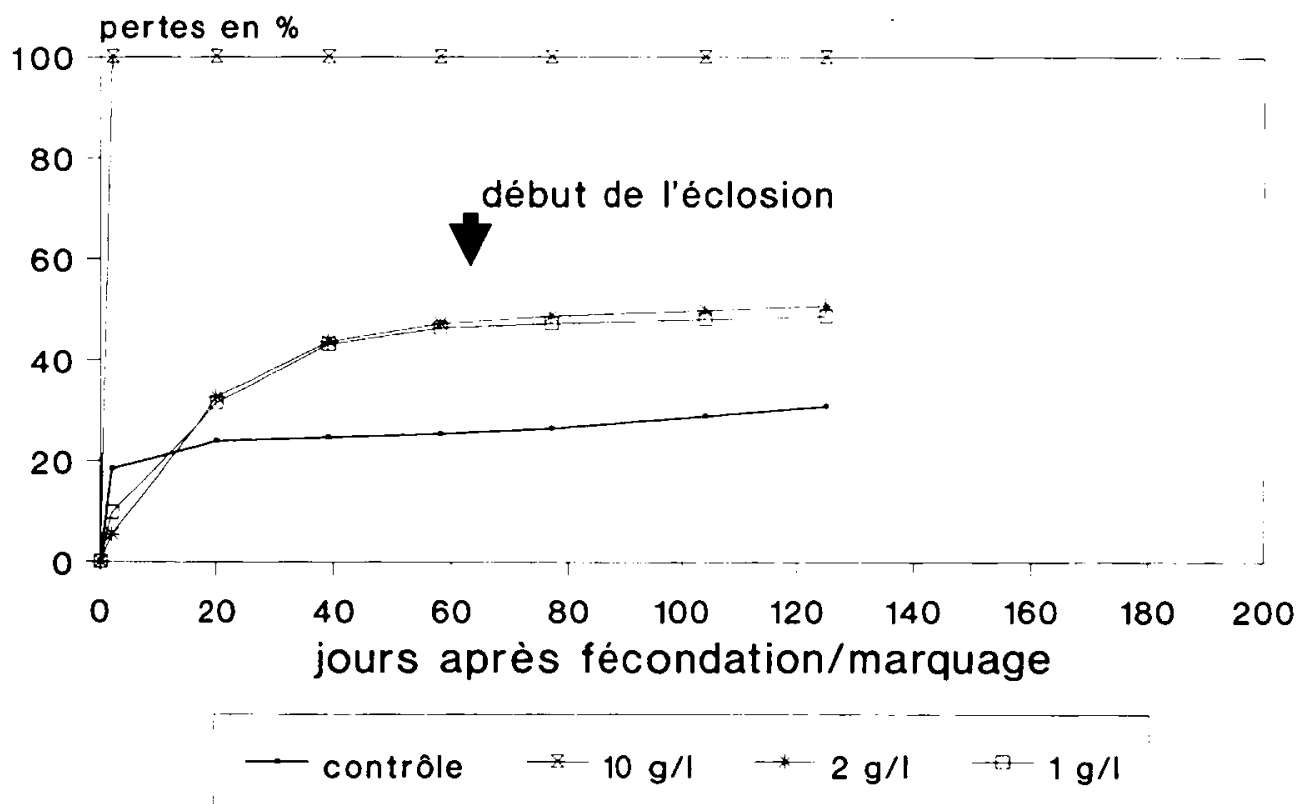

figure 2 : Mortalité pendant l'incubation et l'élevage de truites arc-en-ciel marquées à la tétracycline au moment de la fécondation à différentes concentrations.

figure 2 : Mortality during incubation and hatching of rainbow trouts marked at fertilization with tetracycline at different concentrations. 
Les œufs embryonnés de truite arc-en-ciel traités par balnéation dans une solution de tétracycline à $0.6 \mathrm{~g} / \mathrm{l}$ pendant 14 et 24 heures ont un taux de mortalité au moment de l'éclosion de $15 \%$ tandis que le taux de mortalité est de $32 \%$ pour les œufs non traités.

Le tableau I contient également les pourcentages de poissons marqués de façon visible. Les figures 3 et 4 montrent des otolithes de truites arc-en-ciel marqués au moment de la fécondation, ayant un ou deux nuclei marqués. La figure 5 montre la marque déposée sous forme d'anneau sur un otolithe de truite arc-en-ciel marqué par balnéation de l'œuf embryonné.

\section{DISCusSION}

Les résultats des analyses faites sur les otolithes des cufs traités au moment de la fécondation indiquent que la tétracycline absorbée produit des marques bien visibles si sa concentration est assez élevée. Au microscope à fluorescence, la marque apparaît sous la forme d'une tache ronde orangée (fig. 3) qui correspond exactement au centre de l'otolithe.

D'après les travaux en cours faits sur les corégones (RUHLE et GRIEDER, en prép), l'incorporation du fluorochrome doit se faire dès le début de la calcification des otolithes c'est-à-dire dès le moment où un tissu marquable est à disposition. Cela correspond aux résultats obtenus par MEUNIER et BOIVIN (1974) qui trouvent des marques de tétracycline trois heures après l'injection dans des poissons dont les tissus sont déjà en cours de minéralisation.

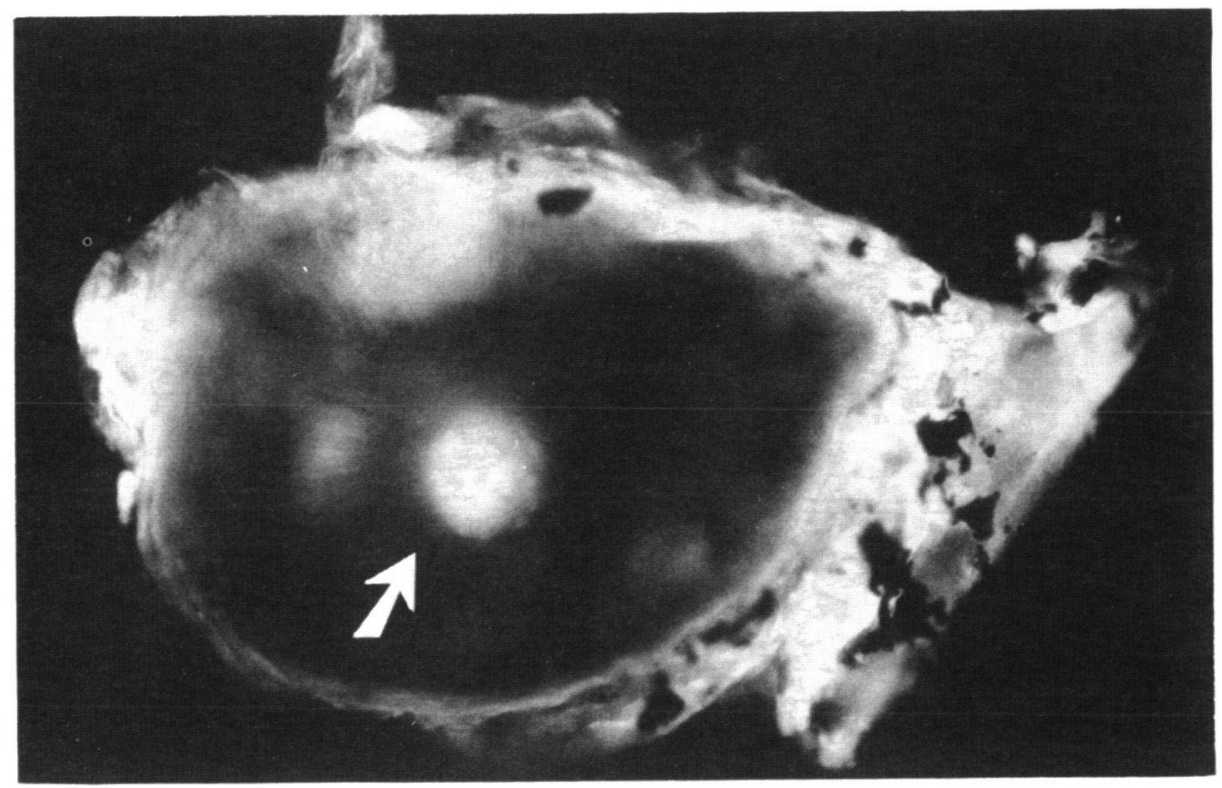

figure 3 : Microphotographie en fluorescence d'un otolithe (sagitta) de truite arc-en ciel marquée par $2 \mathrm{~g} / \mathrm{l}$ de tétracycline pendant $1 \mathrm{~h}$ au moment de la fécondation, montrant 1 nucleus marqué. Truite sacrifiée 155 jours après le marquage, 92 jours après l'éclosion.

Echelle $=100 \mu \mathrm{m}$.

figure 3 : Fluorescence microphotograph of an otolith (sagitta) with one marked nucleus from rainbow trout marked by tetracycline absorption at fertilization. The egg was exposed to $2 \mathrm{~g} / \mathrm{l}$ tetracycline for one hour. Otolith was removed 155 days after marking, 92 days after hatching.

Length of bar $=100 \mu \mathrm{m}$.

Selon GEFFEN (1983), pour les Salmonidés, les nuclei des otolithes apparaissent à 170 degrés-jours après la fécondation. C'est donc à ce moment que la tétracycline commence à être incorporée dans l'otolithe. La formation d'otolithes à partir de plusieurs nuclei telle que GEFFEN l'a observée se manifeste par un marquage de ces différents nuclei (fig. 4). 


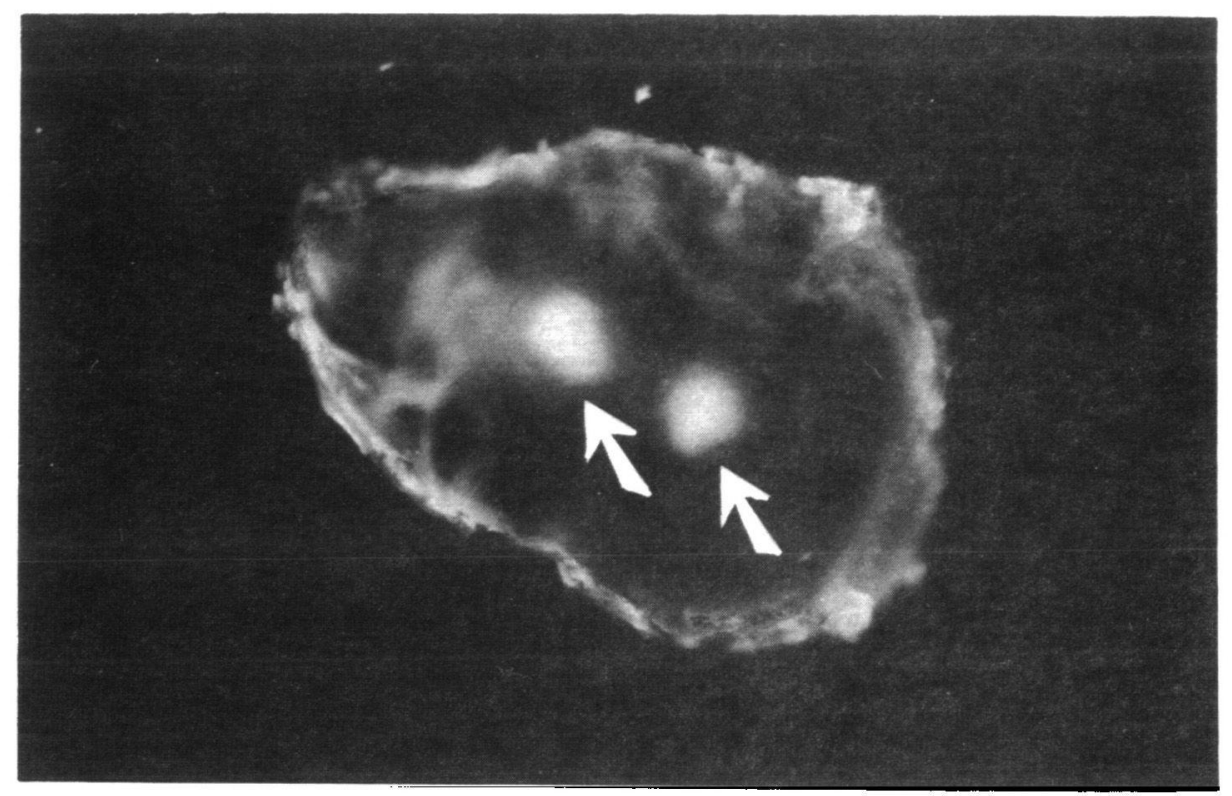

figure 4 : Microphotographle en fluorescence d'un otolithe (sagitta) de truite arc-en-ciel marquée par $2 \mathrm{~g} / \mathrm{l}$ de tétracycline pendant $1 \mathrm{~h}$ au moment de la fécondation, montrant 2 nuclei marqués. Truite sacrifiée 128 jours après le marquage, 65 jours après l'éclosion. Echelle $=100 \mu \mathrm{m}$.

figure 4 : Fluorescence microphotograph of an otolith (sagitta) with two marked nuclei from rainbow trout marked by tetracycline absorption at fertilization. The egg was exposed to $2 \mathrm{~g} / \mathrm{l}$ tetracycline for one hour. Otolith was removed 128 days after marking, 65 days after hatching.

Length of bar $=100 \mu \mathrm{m}$.

Le processus de marquage se poursuit jusqu'à épuisement de la réserve de tétracycline stockée dans le liquide périvitellin. D'après MEUNIER (1982), ce processus a duré de 7 à 10 jours chez la carpe avant que la tétracycline administrée ait été absorbée. Cela indique que l'incorporation du marqueur est continue et lente (SIMMONS et al., 1970).

La visualisation des marques dépend évidemment de la grandeur des poissons observés. Chez de très petits alevins (jusqu'à $25 \mathrm{~mm}$ ), la marque fluorescente se voit directement, les otolithes étant rendus transparents pat l'Entellan. Chez les plus grands, la marque n'est visible sur les sagittae qu'après les avoir amincies et polies, tandis que sur les asterisci, les marques sont encore visibles sans traitement. En observant en premier lieu les asterisci, un amincissement inutile des sagittae peut être évité. L'amincissement est un travail délicat ; il arrive que l'abrasion soit poussée trop loin et mène par conséquent à une interprétation erronée. A partir d'une grandeur de poisson de $40 \mathrm{~mm}$ il est nécessaire d'amincir tous les otolithes. Puisque l'amincissement peut être une source d'erreur et que l'intensité de la marque diffère d'un otolithe à l'autre, l'extraction des deux sagittae et des deux asterisci s'imposait. Dans la mesure du possible, lors d'analyses de stock ou de dy namique de population, il serait préférable d'analyser les otolithes des poissons marqués de moins de $25 \mathrm{~mm}$ afin d'éviter l'abrasion qui demande beaucoup de temps et qui est source d'éventuelles erreurs d'interprétation. Les résultats concernant les pourcentages de poissons marqués de façon visible, résumés dans le tableau I doivent être analysés en considérant ces problèmes de préparation d'otolithes.

Le taux de mortalité des œufs et alevins traités à la tétracycline dépend de la concentration de la solution infiltrée dans l'œuf par osmose avant la fermeture du micropyle. La concentration de la solution doit donc être adaptée à la tolérance des œufs et du sperme.

Des concentrations d'environ $2 \mathrm{~g} / \mathrm{l}$ produisent des marques bien visibles pour les deux espèces sans causer de pertes excessives par rapport à la mortalité naturelle. Il y a, néanmoins, une différence sensible entre le taux de mortalité des truites fario et arc-en-ciel (fig. 1 et 2). Elle est attribuée à une plus grande sensibilité des truites arc-en-ciel envers la tétracycline. La mortalité naturelle, considérée comme élevée, des œufs de truites arc-en-ciel de cette série (contrôle) doit partiellement être liée au fait que les poissons géniteurs se reproduisaient pour la première fois. 
Contrairement à un dépôt de substance marquante formant une tache ronde, le marquage fait par immersion des cufs embryonnés est visible sous forme d'anneau entourant le ou les nuclei et correspondant à la surface de l'otolithe au moment de l'immersion (fig. 5). Cet anneau déposé lors d'une immersion des œufs pendant 14 ou 24 heures reflète la croissance des otolithes pendant ce même laps de temps. La fixation de la tétracycline est par conséquent plus faible, ce qui réduit sensiblement l'intensité de la marque par rapport à la première méthode et rend obligatoire l'amincissement des organes observés. Avec cette deuxième méthode, il faut comme avec la méthode précédente extraire tous les otolithes atteignables, car l'amincissement peut mener à une élimination des marques très minces et à cause de cela exiger l'observation des autres otolithes.

Dans cette expérience, le taux de mortalité était plus faible de $50 \%$ pour les cufs exposés à une solution de tétracycline de concentration relativement faible $(600 \mathrm{mg} / \mathrm{l})$ par rapport aux œufs non traités, ce qui confirmerait l'effet désinfectant de cette substance marquante.

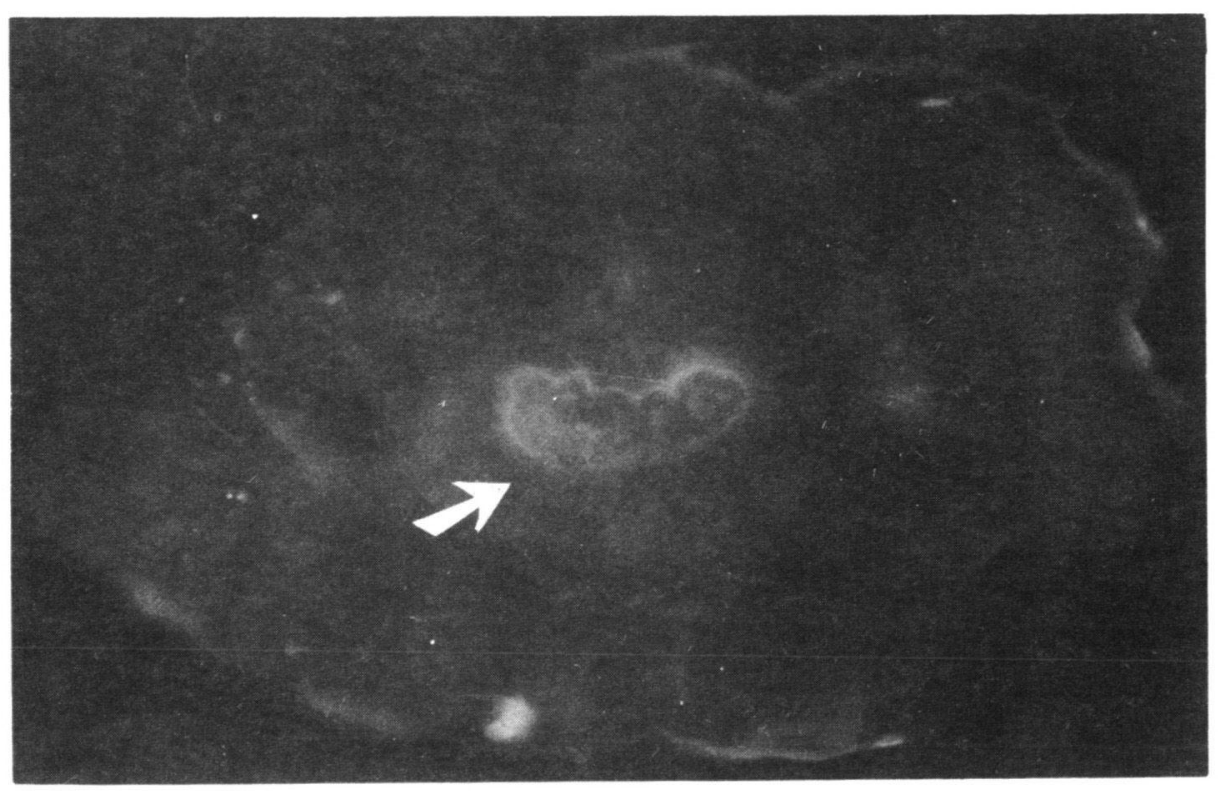

figure 5 : Microphotographie en fluorescence d'un otolithe (sagitta) de truite arc-en-ciel marquée par immersion de l'œuf embryonné dans $600 \mathrm{mg} / \mathrm{l}$ de tétracycline, pendant $24 \mathrm{~h}$. Truite sacrifiée 148 jours après le marquage, 134 jours après l'éclosion. Echelle $=100 \mu \mathrm{m}$.

figure 5 : Fluorescence microphotograph of an otolith (sagitta) from a rainbow trout marked as an eyed egg by exposure to $600 \mathrm{mg} / \mathrm{l}$ tetracycline for $24 \mathrm{~h}$. Otolith was removed 148 days after marking, 134 days after hatching.

Length of bar $=100 \mu \mathrm{m}$.

\section{CONCLUSION}

Le marquage de petits poissons, de larves ou d'œufs embryonnés par immersion dans une solution de tétracycline a pour inconvénient de produire des marques fines difficilement décelables, tout au moins pour les truites utilisées dans cette expérience. Cet inconvénient pourrait être éliminé par une exposition plus longue à concentration plus élevée, ce qui renforcerait le marquage. Ce prolongement du temps d'exposition demande un remplacement fréquent de la solution en raison de son instabilité. Dans le cas d'une expérience à grande échelle, par exemple en bac circulaire, ce remplacement ne pourrait être envisagé pour des raisons financières. Le traitement par la tétracycline au moment de la fécondation marque de façon visible les otolithes et permet de marquer en peu de temps un grand nombre d'œufs à moindre frais. Pour la vérification de l'effet des immersions de petits poissons sur le stock, cette deuxième méthode, conçue il y a dix ans, devrait être prise en considération. Son applicabilité pour des corégones est étudiée (RUHLE et GRIEDER, en prep.) et une expérience comprenant toutes les immersions de Blaufelchen (Coregonus lavaretus (L.)) du lac de Constance est prévue pour 1991. 


\section{REMERCIEMENTS}

Nous remercions Monsieur A. WANDELER de l'Université de Berne pour ses conseils concernant la préparation des otolithes et l'identification des marques de tétracycline.

Nous remercions également Messieurs O. TRUNZ et K. HAUSER pour l'élevage des truites.

\section{BIBLIOGRAPHIE}

DABROWSKI K., TSUKAMOTO K., 1986. Tetracycline tagging in coregonid embryos and larvae. J. Fish Biol., 29, 691-698.

FRY F.E.J., CUCIN D., KENNEDY J.C. et PAPSON A., 1960. The use of lead versenate to place a time mark on fish scales. Trans. Amer. Fish. Soc., 89, 149-153.

GEFFEN A.J., 1983. The deposition of otolith rings in Atlantic Salmon Salmo salar (L.) embryos. J. Fish Biol., 23, 467-474.

HENNICK D.P., TYLER R. W., 1970. Experimental marking of emergent pink salmon Oncorhynchus gorbuscha fry with sprayed fluorescent pigment. Trans. Amer. Fish. Soc., 99, 397-400.

HETTLER W. F., 1984. Marking otoliths by immersion of marine fish larvae in tetracycline. Trans. Amer. Fish. Soc., 113, 370-373.

JONES I. W., 1969. Notes on marking salmon fingerlings with tetracycline. Ann. Prog. Rept. Oregon, Fish. Com., 1-6.

KOBAYASHI S., YUKI R., FURI T., et KOSUGIYAMA T., 1964. Calcification in fish and shellfish. I - Tetracycline labeling patterns of scale, centrum and otolith in young goldfish. Bull. Jap. Soc. Sci. Fish., 30, 6-13.

LORSON D., MUDRAK V.A., 1987. Use of tetracycline to mark otoliths of american shad fry. North Am. J. Fish. Manag., 7, 453-455.

MEUNIER F., 1972. Marquages simples et multiples du tissu osseux de quelques Téléostéens par des substances fluorescentes. C.R. Acad. Sc. Paris, 275, 1.685-1.688.

MEUNIER F., 1974. La technique de marquage vital des tissus squelettiques des poissons. Bull. Fr. Piscic., 225, 52-57.

MEUNIER F., 1982. Étude expérimentale de l'extraction de la tétracycline chez la carpe, Cyprinus carpio L. (Cyprinidae, Téléostéen). Résultats préliminaires. Cybium, 6, (1), 53-64.

MEUNIER F., BOIVIN G., 1972. Marquages multiples du tissu osseux de quelques Téléostéens à l'aide de plusieurs fluorochromes. Bull. Soc. Zool. France, 97, (3), 539-540.

MEUNIER F., BOIVIN G., 1974. Divers aspects de la fixation du chlorhydrate de tétracycline sur les tissus squelettiques de quelques téléostéens. Bull. Soc. Zool. Fr., 99, 495-504.

MEUNIER F., BOIVIN G., 1978. Action de la fluorescéine, de l'alizarine, du bleu de calcéine et de diverses doses de tétracycline sur la croissance de la truite et de la carpe. Ann. Biol. Anim. Biochem. Biophys., 18, (6), 1.293-1.308.

NAGIEC M., DABROWSKI K., NAGIEC C. et MURAWSKA E., 1988. Massmarking of coregonid larvae and fry by tetracycline tagging of otoliths. Aquac. Fish. Manag., 19, 171-178.

NAGIEC M., NAGIEC C., 1983. Marking of juvenile whitefish Coregonus lavaretusL. by tetracycline antibiotics, Rocznik Nauk Rolniczych, Seria H T. 100, 107-114.

NAGIEC M., NAGIEC C., DABROWSKI K. et MURAWSKA E., 1983. Marking of juvenile whitefish Coregonus lavaretus (L.) with tetracycline antibiotics. Acta Ichthyol. Piscat., 13, 47-57.

ODENSE P. H., LOGAN V. H., 1974. Marking Atlantic salmon (Salmo salar) with oxytetracycline. J. Fish. Res. Board Can., 31, 348-350.

SCHMITT P. D., 1984. Marking growth increments in otoliths of larvae and juvenile fish by immersion in tetracycline to examine the rate of increment formation. Fis h. Bull., 82, 237-242.

SCIDMORE W. J., OLSON D. E., 1969. Marking walleye fingerling with oxytetracycline antibiotic. Prog. Fish. Cult., 31, 213-216.

SIMMONS D. J., SIMMONS N. B. et MARSHALL J. H., 1970. The uptake of calcium-45 in the acellular boned toadfish. Calc. Tiss. Res., 5, 206-221.

TROJNAR J. R., 1973. Marking rainbow trout fry with tetracycline. Prog. Fish. Cult., 35, 52-54.

TSUKAMOTO K., 1985. Mass-Marking of Ayu eggs and larvae by tetracycline-tagging of otoliths. Bull. Jap. Soc. Sci. Fish. 51, 903-911.

WEBER D. D., RIDGWAY G. J., 1962. The deposition of tetracycline drugs in bones and scales of fish and its possible use for marking. Prog. Fish Cult., 4, (24), 150-155.

WEBER D., RIDGWAY G. J., 1967. Marking Pacific salmon with tetracycline antibiotics. J. Fish. Res. Bd Canada, 24, (4), 849-865. 